\title{
Locating Creativity in a Framework of Designing for Innovation
}

\author{
John S. Gero ${ }^{1}$ and Udo Kannengiesser ${ }^{2}$ \\ 1 Krasnow Institute for Advanced Study and Volgenau School of \\ Information Technology and Engineering, George Mason University, USA, \\ and University of Technology, Sydney, Australia \\ john@johngero.com \\ 2 NICTA, Australia \\ udo.kannengiesser@nicta.com.au
}

\begin{abstract}
This paper focuses on creativity in the process of designing as the foundation of potential innovations resulting from that process. Using an ontological framework that defines distinct stages in designing, it identifies the locations for creativity independently of their embodiment in human designers or computational tools. The paper shows that innovation, a consequence of creativity, can arise from a large variety of processes in designing.
\end{abstract}

\section{Introduction}

Innovation and creativity are terms that are often used synonymously. This is a result of them sharing the feature of establishing novelty, in the form of new products, systems, processes or organisations [1]. On the other hand, there is an important distinction between them: While creativity generates novel ideas, innovation puts these ideas into practice [2]. This paper focuses on creativity as the foundation and precursor of innovation. This is based on our belief that one of the highest potentials for computer support for design innovation comes from focussing on idea generation rather than only on downstream stages of idea realisation.

We define the notion of novelty, and consequently creativity, relative to the process of designing: Creativity occurs whenever a new design property is introduced for the first time in the ongoing design process, thus changing the state space of possible designs. While there are a number of environment-centric (sociocultural, historic, technological, etc.) factors that determine whether the final product is ultimately accepted as an innovation, our definition of creativity represents a necessary precondition. We will use an ontological framework to locate creativity at various places in the process of designing.

Please use the following format when citing this chapter:

Gero, J. S., Kannengiesser, U., 2007, in IFIP International Federation for Information Processing, Volume 250, Trends in Computer Aided Innovation, ed. León-Rovira, N., (Boston: Springer), pp. 57-66. 


\section{Activities in Creative Designing}

Research in creative designing can be separated into two strands. One strand of research is concerned with developing computational processes that can extend the design state space. Here, five classes of processes have been suggested [3]: emergence, analogy, combination, mutation and first principles. Another strand of research deals with human behaviour involved in changing the state space of possible designs. Here, creativity is viewed as part of more general design behaviour including cognitive and physical activities carried out by the designer.

A paradigm that characterises designing in terms of the activities performed by human designers is situatedness $[4,5,6]$. From the situated perspective, designers perform actions in order to change their environment. By observing and interpreting the results of their actions, they then decide on new actions to be executed. This means that the designers' concepts may change according to what they are "seeing", which itself is a function of what they have done. One may speak of an "interaction of making and seeing" [7] that strongly determines the course of designing by modifying the design state space.

A number of activities related to potential design creativity have been studied that fit with the notion of situatedness. Most of them can be grouped into two classes: interpretation and reflection. Interpretation of design sketches can lead to unexpected discoveries and the invention of new issues or requirements during the design process $[7,8]$. Reflection can bring about similar effects, but is based on internal memory construction rather than external sketches [9]. Both interpretation and reflection are not simple mappings driven by either pure "data push" (replicating previous sketches or memories) or pure "expectation pull" (replicating current expectations). They are rather interactive combinations of both "push" and "pull", and new design concepts emerge from this interaction [10]. Interpretation and reflection are the source of modifications in the design state space. They are orthogonally related to Gero's [3] five computational processes; i.e., any of these processes may occur in any of the two classes of design activities.

\section{An Ontological Framework of Designing}

\subsection{The FBS Ontology}

Gero [11] has proposed an ontology of design objects that provides three high-level categories for the properties of an object: function, behaviour and structure.

Function (F) of an object is defined as its teleology, i.e. "what the object is for". An example is the function "to wake someone up" that humans generally ascribe to the behaviour of an alarm clock.

Behaviour (B) of an object is defined as the attributes that are derived or expected to be derived from its structure, i.e. "what the object does". An example of behaviour is "weight", which can be derived directly from a physical object's structure properties of material and spatial dimensions.

Structure (S) of an object is defined as its components and their relationships, i.e. "what the object consists of". It represents the object's "building blocks" that can be 
directly created or modified by the designer. Examples include molecule structures, mechanical structures, floor and wall structures, and spatial structures.

\subsection{The Situated FBS Framework}

Gero and Kannengiesser [12] have proposed a three-world model of design interactions, Figure 1(a). The external world is composed of representations outside the design agent. The interpreted world is built up inside the design agent in terms of sensory experiences, percepts and concepts. It is the internal representation of that part of the external world that the design agent interacts with. The expected world is the world imagined actions of the design agent will produce. It is the environment in which the effects of actions are predicted according to current goals and interpretations of the current state of the world.

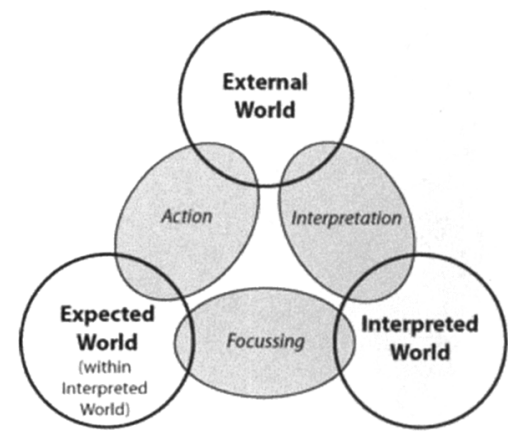

(a)

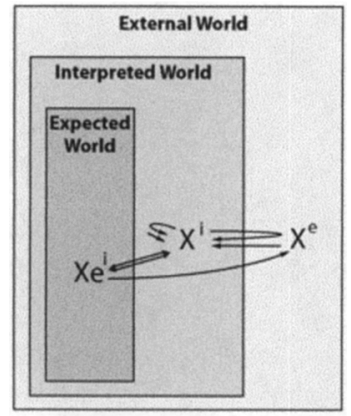

$\mathrm{X}^{\mathrm{e}}=$ external design representation

$\mathrm{X}^{i}=$ interpreted design representation

$\mathrm{Xe}^{\prime}=$ expected design representation

$\longrightarrow=$ action

$\Xi=$ interpretation / reflection

(b)

Fig. 1. Three interacting worlds: (a) general model, (b) specialised model for design representations (after [12]).

These three worlds are linked together by three classes of connections. Interpretation transforms variables which are sensed in the external world into the interpretations of sensory experiences, percepts and concepts that compose the interpreted world. Focussing takes some aspects of the interpreted world, and uses them as goals for the expected world that then become the basis for the suggestion of actions. These actions are expected to produce states in the external world that reach the goals. Action is an effect which brings about a change in the external world according to the goals in the expected world.

Figure 1(b) specialises this model by nesting the three worlds and adding general classes of design representations as well as the activity of reflection [9]. The set of expected design representations ( $\mathrm{Xe}^{\mathrm{i}}$ ) corresponds to the notion of a design state space, i.e. the state space of all possible designs that satisfy the set of requirements. This state space can be modified during the process of designing by transferring new 
interpreted design representations $\left(\mathrm{X}^{\mathrm{i}}\right)$ into the expected world and/or transferring some of the expected design representations $\left(\mathrm{Xe}^{\mathrm{i}}\right)$ out of the expected world. This leads to changes in external design representations $\left(\mathrm{X}^{\mathrm{e}}\right)$, which may then be used as a basis for re-interpretation changing the interpreted world. Novel interpreted design representations $\left(\mathrm{X}^{\mathrm{i}}\right)$ may also be the result of reflection, which can be viewed as a process of interaction among design representations within the interpreted world rather than across the interpreted and the external world. Both interpretation and reflection are represented as push-pull activities.

\section{Locating Creativity in the Situated FBS Framework}

Gero and Kannengiesser's [12] situated FBS framework, Figure 2, combines the FBS ontology with the three-world model. Here, the variable X in Figure 1(b) is replaced with the more specific representations F, B and S. The situated FBS framework also uses explicit representations of external requirements given to the designer. Specifically, there are external requirements on function $\left(\mathrm{FR}^{\mathrm{e}}\right)$, external requirements on behaviour $\left(\mathrm{BR}^{\mathrm{e}}\right)$, and external requirements on structure $\left(\mathrm{SR}^{\mathrm{e}}\right)$.
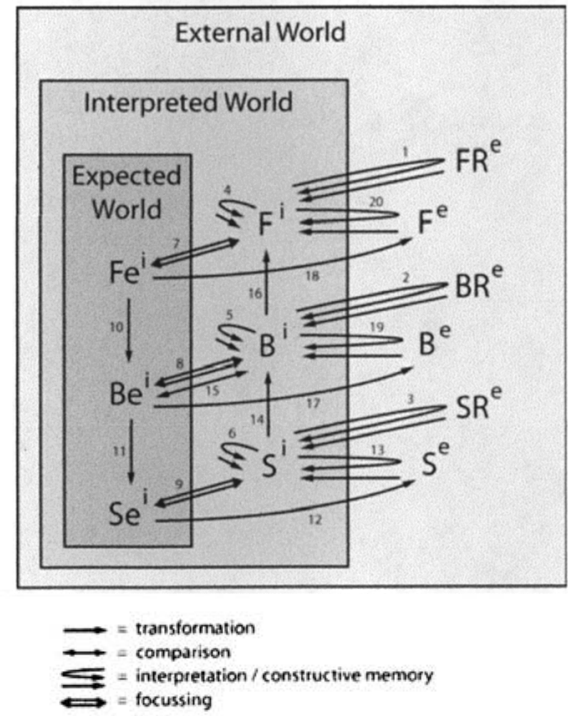

Fig. 2. The situated FBS framework (after [12]).

The situated FBS framework specifies a set of 20 labelled activities that can be mapped them onto eight fundamental design steps [11]. The remainder of this Section will identify potential locations of creativity in each of these steps. 


\subsection{Formulation and Creativity}

Formulation comprises activities 1 to 10 , producing an initial state space of potential design structure, behaviour and function, based on externally given requirements or internal reflection. This design step can already be viewed as a creative act, as it produces a set of design concepts for the first time in the design process. It is based on interpretation and reflection as the generators of new design concepts.

Interpretation (activities 1, 2 and 3) is not a simple reproduction of what is given to the designer. Any two designers will be likely to interpret the same requirements differently, depending on their individual experiences. As a result, they produce different concepts of function, behaviour and structure. This is often based on ambiguities in the requirements given to the designer, especially for function that is often expressed in informal ways.

Reflection (activities 4, 5 and 6) produces additional, implicit requirements that have not been explicitly given to the designer. Cross [13] provides the example of an expert engineer performing the task of designing a device that allows fastening and carrying a backpack on a mountain bike. Based on the engineer's personal experience as a cyclist, an implicit constraint on the structure of the device was constructed, namely to select its location (and thus the location of the backpack) as low as possible for better riding control.

The transformation of function into behaviour (activity 10) can be supported by analogy processes. For example, behaviours of a flexible manufacturing system (FMS) have been specified based on similarities with the functions of an air traffic control (ATC) system [14], Figure 3. Here, functions such as "prevent collisions" and "ensure movements according to the pre-defined plan" are general goals of both systems. Behaviours of one system can then be mapped onto the other.

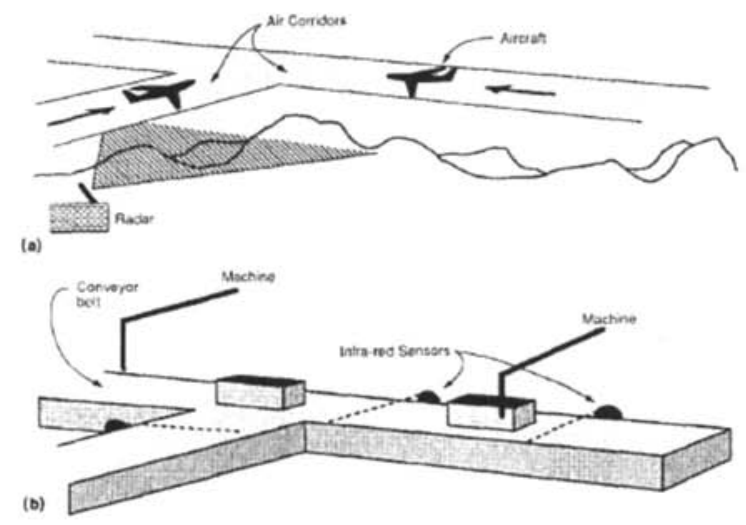

Fig. 3. Key features of the domains of (a) air traffic control and (b) flexible manufacturing (taken from [14]). 


\subsection{Synthesis and Creativity}

Synthesis generates a design solution in terms of a specific instance of structure (activity 11), and subsequently produces an external representation of that instance (activity 12). Synthesis does not modify the design state space, as it uses the concepts produced by formulation without adding new ones, operating only on the values of structure variables rather than on the structure variables themselves. This view excludes a direct association of creativity with the synthesis step. The connection between synthesis and creativity lies in the interpretation of synthesised structure.

\subsection{Analysis and Creativity}

Analysis derives behaviour from synthesised design structure. In the situated FBS framework, this design step is composed of activities 13 and 14. Creativity is generally not desired in analysis, as the primary purpose of this design step is to prepare for the evaluation of the design solution against the pre-determined set of criteria, i.e. within the bounds of the current design state space. However, it is often during the act of analysis that new design issues and ideas emerge that may lead to additions to the design state space. One driver is the interpretation of structure (activity 13), providing the potential for the process of emergence to occur. Examples presented in [15] are concerned with the emergence of shapes through rerepresentation of external structure. The wide range of drafting tools available may support re-representations, such as 2D-, 3D-, "fly-through" and simulation models, each of which can enable the discovery of specific types of design issues.

Emergence during the analysis step is not limited to the structure level. New behaviour often emerges when using computational analysis tools that derive more performances from a given structure than necessary for the currently intended evaluation (activity 14). This is more correctly called supervenience although it is generally taken to be a form of emergence. Take the designer of a material flow system, interested in evaluating the speed of that system. The simulation tool used for the analysis calculates not only speed, but also machine utilisation. This can be seen as the generation of an additional behaviour to be considered in the design state space. Here, creativity is located in the interaction between the designer and the tool.

\subsection{Evaluation and Creativity}

Evaluation assesses the design solution on the basis of the formulated criteria, i.e. by comparison of the behaviour derived from the design solution with the expected behaviour (activity 15). No creativity is involved here.

\subsection{Documentation and Creativity}

Documentation produces an external representation of the final design solution for purposes of communicating that solution (activities 12,17 and 18) to a builder, 
manufacturer or implementer. Creativity plays no role here, because the process of design generation is terminated prior to documentation.

\subsection{Reformulation Type 1 and Creativity}

Reformulation type 1 modifies the structure state space (activity 9). It is based on creative processes that may produce new structure variables. Any of three classes of input is needed for these processes: external requirements on structure, external representations of existing design structure, and interpreted structure representations.

The interpretation of external requirements on structure (activity 3) can bring about new structure variables in two cases. In the first case, modified external requirements are given to the designer after commencement of the design process. Here, the creativity is located in the environment in which the design process is carried out rather than in the design process itself. In the second case, the same external requirements as given at the outset of the design process are interpreted differently. This locates the creativity in the process of interpretation.

The interpretation of external design structure (activity 13) can involve creative processes that generate new structure variables. One example is the process of analogy. Here, an external structure is a source design exhibiting identical behaviour as the current (target) design. The matching and then mapping of the source structure onto the target structure is the creative element of interpretation.

Reflection on interpreted structure (activity 6) constructs new structure variables without the use of external representations. This creative activity is frequently used across the design disciplines to lead the design process into new directions [9]. Reflection on structure is the best-studied type of the reflection processes. For example, the domain of drug design uses reflection on structure to incrementally modify molecules to generate new drugs that meet specified behaviours. Here, reflection is often implemented as crossover (or combination) and mutation processes, embedded in design methodologies using Genetic Algorithms (GAs). GAs take inspiration from biological evolution by applying crossover and mutation operators over populations of candidate structures. Candidates are selected using a fitness function that measures individual performances that correspond to the values of a given behaviour.

\subsection{Reformulation Type 2 and Creativity}

Reformulation type 2 modifies the behaviour state space (activity 8 ). It is based on creative processes that produce new behaviour variables. Any of four classes of input is needed for these processes: external requirements on behaviour, external representations of design behaviour, interpreted behaviour representations, and interpreted structure representations.

The interpretation of external requirements on behaviour (activity 2) can produce new behaviour variables in an analogous way as in reformulation type 1. Creativity is located either outside the system via modified requirements or inside the system via modified interpretation.

The interpretation of external design behaviour (activity 19) can produce new behaviours to alter the behaviour state space. This activity often uses emergence to 
reconceptualise current behaviours, which has mainly been studied in software requirements engineering [16].

Reflection on interpreted behaviour (activity 5) constructs new behaviour variables without the use of any external representations. It reconceptualises current behaviours in a similar way as interpretation. Its underlying mechanism can be assumed to be emergence, although little research has been done here.

The derivation of additional behaviour from structure (activity 14) can drive reformulation type 2 in the same way as described for the analysis step. Creativity here is fostered by the interaction between designers and computational tools. Another way to derive new behaviour from structure is via analogy. Consider the following example: A lamp designer, after coming home from work, looks at a book that still lies half open on his bedside table, Figure 4(a). He becomes aware that the front and back covers of the book can be thought of as defining an arched aperture that can be expanded or reduced by opening or closing the covers. This can be viewed as behaviour derived from an abstraction of the book's structure. The designer realises that in the lighting domain this behaviour could be used as a physical mechanism to fulfil the function of dimming light. He finds this idea interesting and decides to change the design of a desk lamp he is currently working on by implementing this physical dimming mechanism instead of the originally intended electrical one. Figure 4(b) shows a CAD model of the new lamp. It incorporates behaviour and structure features of the book as the source design.

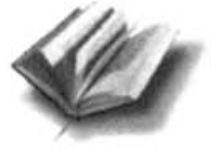

(a)

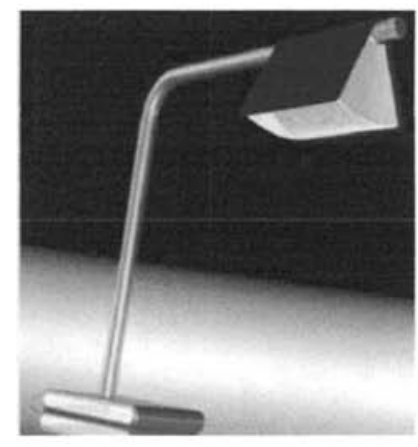

(b)

Fig. 4. Example of analogy in interpretation: (a) a half-open book as a source structure, (b) the desk lamp (model "Hamlet") as the target structure (Source: Stimulo Design).

\subsection{Reformulation Type 3 and Creativity}

Reformulation type 3 modifies the function state space (activity 7). It is based on creative processes that produce new function variables. Any of four classes of input is needed for these processes: external requirements on function, external representations of design function, interpreted function representations, and interpreted behaviour representations. 
The interpretation of external requirements on function (activity 1) can produce new function variables in an analogous way as in reformulation type 1 and 2 . Creativity is located either outside the system via modified requirements or inside the system via modified interpretation.

The interpretation of external design function (activity 20) can produce new functions to alter the function state space. Maiden et al. [17] have described how designers of air traffic management software combined existing software functions into new ones during a creativity workshop. Here, the two functions "to allow air traffic controllers to maintain an accurate mental model of the air space" and "to offer new types of situational display to air traffic controllers" were combined into the new function "to allow air traffic controllers to rewind and fast-forward aircraft movements to develop their mental models of the air space before taking responsibilities for decisions that they will make".

Reflection on interpreted function (activity 4) constructs new functions without the use of any external representations. Little research has been done in this area.

The derivation of function from behaviour (activity 16) can result in new functions to be incorporated in the design. Schön [18] has presented the example of Scotch Tape, whose initial function was "to mend books". However, the people buying this product invented additional functions, such as "to wrap packages" and "to curl hair". These functions have been likely to be derived from the behaviour of removable adhesiveness. They subsequently led to adaptations of the product to different applications such as packaging and hair curling.

\section{Conclusion}

We have shown in this paper that the situated FBS framework provides an ontological basis for locating creativity at various stages in designing. While the three classes of reformulation are the design steps that include most creative activities, the framework can locate creativity also at other places in the design process.

Most importantly, our framework provides the foundations for developing better computational tools to support creativity and innovation. This is based on the independence of an ontological view of creativity with respect to its embodiment. All creative processes and activities in designing can be located in human designers, their tools and the interaction between the designers and the tools.

Innovation is rooted in creativity. Whilst creativity is the process of generating new designs, innovation is the process of putting novel designs into practice. It requires a knowledge of what is novel in terms of function, behavior and structure to be able to demonstrate that the design is novel in at least one of those dimensions. An ontological framework of designing allows both the locus of creativity to be determined and the support for innovation to be articulated. 


\section{Acknowledgements}

This research is supported by a grant from the Australian Research Council, grant no. DP0559885 - Situated Design Computing. It was carried out at the Key Centre of Design Computing and Cognition, University of Sydney. The authors wish to thank the company Stimulo Design for providing the example of the "Hamlet" desk lamp.

\section{References}

1. J. Fagerberg, D.C. Mowery and R.R. Nelson, The Oxford Handbook of Innovation (Oxford University Press, Oxford, 2005).

2. D. Gurteen, Knowledge, creativity and innovation, Journal of Knowledge Management 2(1), 5-13 (1998).

3. J.S. Gero, Creativity, emergence and evolution in design, Knowledge-Based Systems 9(7), 435-448 (1996).

4. J. Dewey, The reflex arc concept in psychology, Psychological Review 3, 357-370 (1896 reprinted in 1981).

5. F.C. Bartlett, Remembering: A Study in Experimental and Social Psychology (Cambridge University Press, Cambridge, 1932 reprinted in 1977).

6. W.J. Clancey, Situated Cognition: On Human Knowledge and Computer Representations (Cambridge University Press, Cambridge, 1997).

7. D.A. Schön and G. Wiggins, Kinds of seeing and their functions in designing, Design Studies 13(2), 135-156 (1992).

8. M. Suwa, J.S. Gero and T. Purcell, Unexpected discoveries and s-inventions of design requirements: A key to creative designs, in: Computational Models of Creative Design IV, edited by J.S. Gero and M.L. Maher (Key Centre of Design Computing and Cognition, University of Sydney, Sydney, Australia, 1999), pp. 297-320.

9. D.A. Schön, Educating the Reflective Practitioner: Toward a New Design for Teaching and Learning in the Professions (Jossey-Bass Publishers, San Francisco, 1987).

10. J.S. Gero and H. Fujii, A computational framework for concept formation for a situated design agent, Knowledge-Based Systems 13(6), 361-368 (2000).

11. J.S. Gero, Design prototypes: A knowledge representation schema for design, $A I$ Magazine 11(4), 26-36 (1990).

12. J.S. Gero and U. Kannengiesser, The situated function-behaviour-structure framework, Design Studies 25(4), 373-391 (2004).

13. N. Cross, Creative cognition in design: Processes of exceptional designers, in: Creativity and Cognition'02, edited by T. Hewett and T. Kavanagh (ACM Press, New York, 2002), pp. 14-19.

14. N.A. Maiden and A.G. Sutcliffe, Exploiting reusable specifications through analogy, Communications of the ACM 35(4), 55-63 (1992).

15. H.J. Jun and J.S. Gero, Representation, re-representation and emergence in collaborative computer-aided design, in: Preprints Formal Aspects of Collaborative Computer-Aided Design, edited by M.L. Maher, J.S. Gero and F. Sudweeks (Key Centre of Design Computing and Cognition, University of Sydney, Australia, 1997), pp. 303-320.

16. L. Nguyen and P.A. Swatman, Managing the requirements engineering process, Requirements Engineering 8(1), 55-68 (2003).

17. N.A. Maiden, A. Gizikis and S. Robertson, Provoking creativity: Imagine what your requirements could be like, IEEE Software 21(5), 68-75 (2004).

18. D.A. Schön, 1983, The Reflective Practitioner: How Professionals Think in Action (Harper Collins, New York, 1983). 